\title{
Boundedness of the extremal solution of some $p$-Laplacian problems
}

\author{
Manel Sanchón
}

Centro de Matemática, Universidade de Coimbra, 3001-454 Coimbra, Portugal

Received 6 February 2006; accepted 31 May 2006

\begin{abstract}
In this article we consider the $p$-Laplace equation $-\Delta_{p} u=\lambda f(u)$ on a smooth bounded domain of $\mathbb{R}^{N}$ with zero Dirichlet boundary conditions. Under adequate assumptions on $f$ we prove that the extremal solution of this problem is in the energy class $W_{0}^{1, p}(\Omega)$ independently of the domain. We also obtain $L^{q}$ and $W^{1, q}$ estimates for such a solution. Moreover, we prove its boundedness for some range of dimensions depending on the nonlinearity $f$.
\end{abstract}

(c) 2006 Elsevier Ltd. All rights reserved.

MSC: $35 \mathrm{~J} 70 ; 35 \mathrm{~J} 60$

Keywords: p-Laplacian; Extremal solution; Regularity

\section{Introduction}

Let $\Omega$ be a smooth bounded domain of $\mathbb{R}^{N}$ and $p>1$. We consider the following Dirichlet problem for the $p$-Laplacian operator $-\Delta_{p} u:=-\operatorname{div}\left(|\nabla u|^{p-2} \nabla u\right)$,

$$
\begin{cases}-\Delta_{p} u=\lambda f(u) & \text { in } \Omega, \\ u=0 & \text { on } \partial \Omega,\end{cases}
$$

where $\lambda$ is a positive parameter and $f$ satisfies the following assumptions:

$f$ is an increasing $C^{2}$ function such that $f(0)>0, f(t)^{1 /(p-1)}$ is

superlinear at infinity (i.e., $f(t) / t^{p-1} \rightarrow+\infty$ as $\left.t \rightarrow+\infty\right)$,

\footnotetext{
This work was partially supported by CMUC/FCT and MCyT grants BMF2002-04613-C03, MTM2005-07660-C02.

E-mail address: msanchon@mat.uc.pt.
} 
and

$$
(f(t)-f(0))^{1 /(p-1)} \text { is convex in }[0,+\infty) .
$$

We say that $u \in W_{0}^{1, p}(\Omega)$ is a solution of $\left(1_{\lambda, p}\right)$ if $f(u) \in L^{1}(\Omega)$ and

$$
\int_{\Omega}|\nabla u|^{p-2} \nabla u \cdot \nabla \varphi \mathrm{d} x=\lambda \int_{\Omega} f(u) \varphi \mathrm{d} x, \quad \text { for all } \varphi \in C_{0}^{1}(\Omega) .
$$

Such solutions are usually known as weak energy solutions. For short, we will refer to them simply as solutions.

On the other hand, we say that $u \in W_{0}^{1, p}(\Omega)$ is a regular solution of $\left(1_{\lambda, p}\right)$ if $f(u) \in L^{\infty}(\Omega)$ and satisfies (4). Using regularity results for degenerate elliptic equations, one has that every regular solution belongs to $C^{1, \alpha}(\bar{\Omega})$ for some $\alpha>0$ (see [7,22], and [17]).

Under assumption (2), Cabré and the author [5] proved the existence of an extremal parameter $\lambda^{*} \in(0, \infty)$ such that: if $\lambda<\lambda^{*}$ then problem $\left(1_{\lambda, p}\right)$ admits a regular solution $u_{\lambda}$ which is minimal among all other possible solutions, and if $\lambda>\lambda^{*}$ then problem $\left(1_{\lambda, p}\right)$ admits no regular solution. Moreover, minimal solutions are semi-stable in the sense that the second variation of the energy functional associated with $\left(1_{\lambda, p}\right)$ is nonnegative definite (see Definition 8 below). Using this property [5] establishes that

$$
u^{*}:=\lim _{\lambda \uparrow \lambda^{*}} u_{\lambda}
$$

is a solution of $\left(1_{\lambda^{*}, p}\right)$ whenever the nonlinearity $f(u)$ makes its growth comparable to $u^{m} ; u^{*}$ is called the extremal solution. As a particular case, the power nonlinearity $f(u)=(1+u)^{m}$ with $m>p-1$ is studied; we obtain that $u^{*}$ is a bounded (and hence regular) solution if

$$
N<G(m, p):=\frac{p}{p-1}\left(1+\frac{m p}{m-(p-1)}+2 \sqrt{\frac{m}{m-(p-1)}}\right) .
$$

Ferrero [9] also obtained (independently of [5]) the boundedness of the extremal solution when $N<G(m, p)$ and proved using phase plane techniques that $u^{*}$ is unbounded if $N \geq G(m, p)$ and the domain $\Omega$ is the unit ball of $\mathbb{R}^{N}$.

García-Azorero, Peral, and Puel [11,12] studied in detail problem $\left(1_{\lambda, p}\right)$ when $f(u)=e^{u}$. They proved that $u^{*}$ is a solution independently of $\Omega$, and that $u^{*}$ is a bounded solution if in addition

$$
N<F(p):=p+\frac{4 p}{p-1} .
$$

Moreover, if $N \geq p+4 p /(p-1)$ and the domain $\Omega$ is the unit ball of $\mathbb{R}^{N}$ then $u^{*}$ is unbounded. All these results were first obtained for the Laplacian problem $\left(1_{\lambda, 2}\right)$. Crandall and Rabinowitz [6] obtained the existence of the branch of minimal solutions $\left\{\left(\lambda, u_{\lambda}\right): \lambda \in\left(0, \lambda^{*}\right)\right\}$ and proved that $u^{*}$ is a solution of the extremal problem $\left(1_{\lambda^{*}, 2}\right)$ for the exponential and power nonlinearities. Moreover, they proved the boundedness of the extremal solution in the range of dimensions commented on before (for $p=2$ ). Joseph and Lundgren [14] made a detailed analysis for both nonlinearities when the domain is the unit ball of $\mathbb{R}^{N}$. Using phase plane techniques, they obtained that $u^{*}$ is an unbounded solution if $N \geq G(m, 2)$ for $f(u)=(1+u)^{m}$, and if $N \geq F(2)=10$ for $f(u)=e^{u}$, where $G$ and $F$ are defined in (6) and (7), respectively. Brezis et al. [2] proved, under assumptions (2) and (3), that $u^{*}$ is a weak solution of $\left(1_{\lambda^{*}, 2}\right)$. Moreover, they proved nonexistence results for $\lambda>\lambda^{*}$. Brezis and Vázquez [3] gave a characterization of 
singular semi-stable solutions and, as consequence, obtained the results in [14] using variational methods instead of phase plane techniques. In [19,10,18,23], and [8] other results can be found about the extremal solution of problem $\left(1_{\lambda^{*}, 2}\right)$.

In [21] it is proved, assuming only (2), (3), and $p \geq 2$, that $u^{*}$ is a solution of $\left(1_{\lambda^{*}, p}\right)$ if $N<p\left(1+p^{\prime}\right)$, where $p^{\prime}=p /(p-1)$. Moreover, if $p+p^{\prime} \leq N<p\left(1+p^{\prime}\right)$ then $u^{*} \in L^{q}(\Omega)$, for all $1 \leq q<\overline{q_{0}}$, and $u^{*} \in W_{0}^{1, q}(\Omega)$, for all $1 \leq q<\bar{q}_{1}$, where

$$
\bar{q}_{0}:=(p-1) \frac{N}{N-\left(p+p^{\prime}\right)} \quad \text { and } \quad \bar{q}_{1}:=(p-1) \frac{N}{N-\left(1+p^{\prime}\right)} .
$$

It is also proved that $u^{*} \in L^{\infty}(\Omega)$ if $N<p+p^{\prime}$. These results extend a work due to Nedev [20] for $p=2$, establishing that $u^{*}$ is a solution if $N \leq 5$, and that $u^{*}$ is bounded if $N \leq 3$. It is still an open problem to prove the boundedness (or not) of the extremal solution when $p\left(1+p^{\prime}\right) \leq N<F(p)=p+4 p^{\prime}$ even for $p=2$ (note that when $f(u)=e^{u}$ and the domain $\Omega$ is the unit ball of $\mathbb{R}^{N}, u^{*}$ is an unbounded solution if $N \geq F(p)$ ).

The main results of this work use the semi-stability property of minimal solutions to establish the boundedness of the extremal solution for a large class of nonlinearities. The first one applies to every convex $f$ when $1<p<2$ and to some convex $f$ when $p=2$.

Theorem 1. Assume (2) and (3). Let $u^{*}$ be the function defined in (5). The following assertions hold:

(i) If $f$ is a convex function, $1<p<2$, and

$$
N \leq H(p):=p+\frac{2 p}{p-1}(1+\sqrt{2-p}),
$$

then $u^{*}$ is a regular solution of $\left(1_{\lambda^{*}, p}\right)$. In particular, $u^{*} \in L^{\infty}(\Omega)$.

(ii) Let

$$
\tau_{-}:=\liminf _{t \rightarrow+\infty} \frac{(f(t)-f(0)) f^{\prime \prime}(t)}{f^{\prime}(t)^{2}} .
$$

If $p=2,0<\tau_{-}$, and $N \leq 6$, then $u^{*}$ is a regular solution of $\left(1_{\lambda^{*}, 2}\right)$. In particular, $u^{*} \in L^{\infty}(\Omega)$.

First, we note that part (ii) extends the main result in [20] under an additional assumption on $f: 0<\tau_{-}$. Second, as we said before, if $N \geq F(p)$, where $F$ is defined in (7), then the extremal solution $u^{*}$ is not necessarily bounded. Since $1<F(p)-H(p)<4$, for all $1<p<2$, the optimal or larger dimension ensuring the boundedness will differ from (8) at most by four.

The next result extends Theorem 1 , and gives $L^{q}$ and $W_{0}^{1, q}$ estimates for the extremal solution of $\left(1_{\lambda^{*}, p}\right)$. Its proof uses some of the arguments appearing in [20] and [21].

Theorem 2. Assume (2) and (3). Let $u^{*}$ and $\tau_{-}$be defined in (5) and (9), respectively. If

$$
\frac{p-2}{p-1}<\tau_{-}
$$

then $u^{*}$ is a solution of $\left(1_{\lambda^{*}, p}\right)$. Moreover the following assertions hold:

(i) If in addition

$$
N<N(p):=p+\frac{2 p}{p-1}\left(1+\sqrt{1-(p-1)\left(1-\tau_{-}\right)}\right),
$$

then $u^{*} \in L^{\infty}(\Omega)$. 
(ii) If in addition $N \geq N(p)$ then $u^{*} \in L^{q}(\Omega)$, for all $1 \leq q<q_{0}$, and $u^{*} \in W_{0}^{1, q}(\Omega)$, for all $1 \leq q<q_{1}$, where

$$
q_{0}:=\frac{\left(p+2 \sqrt{1-(p-1)\left(1-\tau_{-}\right)}\right) N}{N-N(p)}
$$

and

$$
q_{1}:=\frac{(p-1)\left(p+2 \sqrt{1-(p-1)\left(1-\tau_{-}\right)}\right) N}{(p-1) N-2\left(p+\sqrt{1-(p-1)\left(1-\tau_{-}\right)}\right)} .
$$

For $f(u)=e^{u}$ we have that $\tau_{-}=1$ and hence $N(p)=F(p)$, where $F$ is defined in (7). Therefore, Theorem 2(i) recovers the boundedness of the extremal solution for the exponential nonlinearity. It also extends the main results in [21] under the assumption (10). However, $(p-2) /(p-1) \leq \tau_{-}$whenever (3) holds. Indeed, defining $h(t):=(f(t)-f(0))^{1 /(p-1)}$ and using (3) one obtains that $h^{\prime \prime}(t) \geq 0$ for all $t \geq 0$, or equivalently,

$$
\frac{(f(t)-f(0)) f^{\prime \prime}(t)}{f^{\prime}(t)^{2}} \geq \frac{p-2}{p-1} \text { for all } t \geq 0 .
$$

Finally, it is easy to check that (10) implies the existence of positive constants $c$ and $m>p-1$ such that $f(t) \geq c(1+t)^{m}$ for all $t \geq 0$. Hence, we are assuming more than the superlinearity of $f(t)^{1 /(p-1)}$ at infinity.

Theorem 2(i) applied to $f(u)=(1+u)^{m}$ with $m>p-1$ does not recover the results commented on before. Using Lemma 3.2 in [5] we improve Theorem 2 for some reaction terms $f(u)$ that make its growth comparable to a power of $u$.

Theorem 3. Assume (2), (3), and that there exist positive constants $m$ and $c$ such that

$$
0 \leq f(t) \leq c(1+t)^{m}, \quad \text { for all } t \geq 0 .
$$

Let $u^{*}$ and $\tau_{-}$be defined in (5) and (9), respectively. If $(p-2) /(p-1)<\tau_{-}$and

$$
N<\frac{p}{p-1}\left(1+\frac{m p}{m-(p-1)}+\frac{2 m \sqrt{1-(p-1)\left(1-\tau_{-}\right)}}{m-(p-1)}\right),
$$

then $u^{*}$ is a regular solution of $\left(1_{\lambda^{*}, p}\right)$. In particular, $u^{*} \in L^{\infty}(\Omega)$.

For $f(u)=(1+u)^{m}$ with $m>p-1$, we have

$$
\frac{p-2}{p-1}<\tau_{-}=\frac{m-1}{m} \text {. }
$$

Therefore, by Theorem 3 applied to $f(u)=(1+u)^{m}$ with $m>p-1$, we obtain that $u^{*} \in L^{\infty}(\Omega)$ if $N<G(m, p)$, where $G$ is defined in (6). As a consequence, this result is optimal for the pure power nonlinearity.

Our last theorem takes into account the number

$$
\tau_{+}:=\limsup _{t \rightarrow+\infty} \frac{(f(t)-f(0)) f^{\prime \prime}(t)}{f^{\prime}(t)^{2}} .
$$


Point (i) will be proved using the fact that $\tau_{+}<1$ implies the existence of positive constants $m$ and $c$ such that (13) holds (see Lemma 13 below), and then Theorem 3. Point (ii) is an immediate consequence of Theorem 2(i). Point (iii) follows from parts (i) and (ii).

Theorem 4. Assume (2) and (3). Let $u^{*}, \tau_{-}$, and $\tau_{+}$be defined in (5), (9) and (15), respectively. If $\tau_{-}>(p-2) /(p-1)$ then $u^{*}$ is a solution of $\left(1_{\lambda^{*}, p}\right)$. Moreover the following assertions hold: (i) Assume $\tau_{+}<1$. If in addition

$$
N<\frac{p}{p-1}\left(1+\frac{p}{1-(p-1)\left(1-\tau_{+}\right)}+\frac{2 \sqrt{1-(p-1)\left(1-\tau_{-}\right)}}{1-(p-1)\left(1-\tau_{+}\right)}\right),
$$

then $u^{*} \in L^{\infty}(\Omega)$.

(ii) Assume $\tau_{+} \geq 1$. If in addition

$$
N<N(p)=p+\frac{2 p}{p-1}\left(1+\sqrt{1-(p-1)\left(1-\tau_{-}\right)}\right),
$$

then $u^{*} \in L^{\infty}(\Omega)$.

(iii) Assume $\tau_{-}=\tau_{+}$. If in addition

$$
N<F(p)=p+\frac{4 p}{p-1},
$$

then $u^{*} \in L^{\infty}(\Omega)$.

We remark that part (iii) in this theorem is sharp in the sense that there exists a nonlinearity $f$ and a domain $\Omega$ such that the extremal solution $u^{*}$ is unbounded if $N \geq F(p)$. Recently, Cabré, Capella, and the author [4] proved, when $\Omega$ is the unit ball of $\mathbb{R}^{N}$ and $f$ is a general locally Lipschitz function, the boundedness of the extremal solution if $N<F(p)$. As we said before, this fact remains open for general domains. Theorem 4 gives a positive answer to this question for some nonlinearities.

Finally, we note that in all our results we are assuming $(p-2) /(p-1)<\tau_{-}$. Using the a priori estimates obtained in [21] and Lemma 3.2 in [5], it is possible to obtain analogous regularity results when $\tau_{-}=(p-2) /(p-1)$ and (13) (or $\left.\tau_{+}<1\right)$ holds. For instance, it can be proved that $u^{*}$ is bounded for all $N$ if $\tau=\tau_{-}=\tau_{+}=(p-2) /(p-1)$. By Theorem 4 , one expects to obtain the last assertion, since the function appearing in the right-hand side of (16) tends to infinity as $\tau$ goes to $(p-2) /(p-1)$.

The paper is organized as follows. In Section 2 we give some known results. In Section 3, we prove the existence and regularity of the extremal solution under suitable hypotheses on $f$ which include the assumptions in Theorems 1 and 2 (see Proposition 10 below). In Section 4 we prove Theorems 1 and 2. Finally, in Section 5, we prove Theorems 3 and 4.

\section{Known results}

We consider

$$
\begin{cases}-\Delta_{p} u=g(x) & \text { in } \Omega, \\ u=0 & \text { on } \partial \Omega,\end{cases}
$$

where $g \in L^{q}(\Omega)$ for some $q \geq 1$.

The following result can be found in [13] or in [1]. 
Lemma 5. Assume that $g \in L^{q}(\Omega)$, for some $q \geq 1$, and that $u$ is a solution of (18). The following assertions hold:

(i) If $q>N / p$ then $u \in L^{\infty}(\Omega)$. Moreover,

$$
\|u\|_{\infty} \leq C\|g\|_{q}^{\frac{1}{p-1}}
$$

where $C$ is a constant depending only on $N, p, q$, and $|\Omega|$.

(ii) If $q=N / p$ then $u \in L^{r}(\Omega)$ for all $1 \leq r<+\infty$. Moreover,

$$
\|u\|_{r} \leq C\|g\|_{q}^{\frac{1}{p-1}}
$$

where $C$ is a constant depending only on $N, p, r$, and $|\Omega|$.

(iii) If $1 \leq q<N / p$ then $|u|^{r} \in L^{1}(\Omega)$ for all $0<r<r_{1}$, where $r_{1}:=(p-1) N q /(N-q p)$. Moreover,

$$
\left\||u|^{r}\right\|_{1}^{1 / r} \leq C\|g\|_{q}^{\frac{1}{p-1}},
$$

where $C$ is a constant depending only on $N, p, q, r$, and $|\Omega|$.

To obtain the estimates for the gradient of the extremal solution we will use the following regularity result which follows from Theorem 1.6 in [15].

Lemma 6. If $g \in L^{q}(\Omega)$ for some $q \geq \tilde{q}$, where

$$
\tilde{q}:=\frac{N p}{(p-1) N+p},
$$

then there exists a unique solution $u$ of (18). If in addition $q<N / p$, then $u \in W_{0}^{1, r}(\Omega)$, where $r=(p-1) N q /(N-q)$.

Remark 7. We note that the existence and uniqueness of a solution is well known if $f \in$ $W^{-1, p^{\prime}}(\Omega)$ (see [16]), and hence, if $f \in L^{\tilde{q}}(\Omega)$ (since $\tilde{q}=\left(p^{*}\right)^{\prime}$, where $p^{*}=N p /(N-p)$ corresponds to the critical Sobolev embedding).

Now, we recall the definition of semi-stable solution introduced in [5] and give a technical lemma that we will use to prove Theorem 3 (see Lemma 3.2 in [5]).

Definition 8. Let $u \in W_{0}^{1, p}(\Omega)$ be a solution of $\left(1_{\lambda, p}\right)$. Define

$$
A_{u}:=W_{0}^{1, p}(\Omega) \text { if } p \geq 2,
$$

and

$$
\begin{gathered}
A_{u}:=\left\{\psi \in W_{0}^{1, p}(\Omega):|\psi| \leq C u \text { and }|\nabla \psi| \leq C|\nabla u| \text { in } \Omega, \text { for some constant } C\right\} \\
\text { if } 1<p<2 .
\end{gathered}
$$

We say that $u$ is semi-stable if

$$
\int_{\{\nabla u \neq 0\}}|\nabla u|^{p-2}\left\{(p-2)\left(\frac{\nabla u}{|\nabla u|} \cdot \nabla \psi\right)^{2}+|\nabla \psi|^{2}\right\} \mathrm{d} x-\lambda \int_{\Omega} f^{\prime}(u) \psi^{2} \mathrm{~d} x \geq 0,
$$

for all $\psi \in A_{u}$. 
We note that the left-hand side of (20) is the second variation of the energy functional associated with $\left(1_{\lambda, p}\right)$ and that it is well defined on the set of admissible functions $A_{u}$ (see [5] for more comments).

Lemma 9. Assume that there exist positive constants $m$ and $c$ such that

$$
0 \leq f(t) \leq c(1+t)^{m}, \quad \text { for all } t \geq 0 .
$$

Let $u$ be a solution of $\left(1_{\lambda, p}\right)$. If $f(u) \in L^{q}(\Omega)$ for some $q \geq 1$ satisfying

$$
\left(1-\frac{p-1}{m}\right) N<q p
$$

then

$$
\|u\|_{\infty} \leq C,
$$

where $C$ is a constant depending only on $N, m, p, q,|\Omega|, c$, and $\|\lambda f(u)\|_{q}$.

\section{Preliminaries}

The proof of all the results stated in the introduction is based in the following proposition.

Proposition 10. Assume (2) and (3), and define $\tilde{f}(t):=f(t)-f(0)$. If there exists $\gamma \geq 1 /(p-1)$ such that

$$
\limsup _{t \rightarrow+\infty}(p-1) \gamma^{2} \frac{\int_{0}^{t} \tilde{f}(s)^{2 \gamma-2} f^{\prime}(s)^{2} \mathrm{~d} s}{\tilde{f}(t)^{2 \gamma-1} f^{\prime}(t)}<1,
$$

then $u^{*}=\lim _{\lambda \uparrow \lambda^{*}} u_{\lambda}$ is a solution of $\left(1_{\lambda^{*}, p}\right)$. Moreover, the following assertions hold:

(i) If $N<(2 \gamma+1) p$ then $u^{*} \in L^{\infty}(\Omega)$. In particular $f\left(u^{*}\right) \in L^{\infty}(\Omega)$.

(ii) If $N \geq(2 \gamma+1) p$ then $u^{*} \in L^{q}(\Omega)$, for all $1 \leq q<\tilde{q}_{0}$, and $f\left(u^{*}\right) \in L^{q}(\Omega)$, for all $1 \leq q<\tilde{q}_{1}$, where

$$
\tilde{q}_{0}:=\frac{((p-1)(2 \gamma+1)-1) N}{N-(2 \gamma+1) p} \quad \text { and } \quad \tilde{q}_{1}:=\frac{(2 \gamma+1-1 /(p-1)) N}{N-p /(p-1)} \text {. }
$$

Remark 11. First, we note that for $N=(2 \gamma+1) p$, we have $\tilde{q_{0}}=+\infty$ and hence, in this case, one obtains that $u^{*} \in L^{q}(\Omega)$ for all $1 \leq q<+\infty$.

On the other hand, we want to explain the relation between assumptions (3) and (21). Let $h(t)=\tilde{f}(t)^{1 /(p-1)}$. By (3), $h$ is a convex function in $[0,+\infty)$. In particular, $h^{\prime}(t) \geq h(t) / t$ for all $t>0$, or equivalently,

$$
f^{\prime}(t) \geq(p-1) \frac{\tilde{f}(t)}{t}, \quad \text { for all } t>0 .
$$

Therefore, under assumption (2), we obtain that $f^{\prime}(t)>0$ for all $t>0$.

Moreover, since $h^{\prime}(s) \leq h^{\prime}(t)$, for all $0<s<t$, we have

$$
f^{\prime}(s) \leq\left(\frac{\tilde{f}(t)}{\tilde{f}(s)}\right)^{\frac{2-p}{p-1}} f^{\prime}(t), \quad \text { for all } 0<s<t .
$$


From this inequality, we obtain

$$
\int_{0}^{t} \tilde{f}(s)^{2 \gamma-2} f^{\prime}(s)^{2} \mathrm{~d} s \leq\left(2 \gamma-\frac{1}{p-1}\right)^{-1} \tilde{f}(t)^{2 \gamma-1} f^{\prime}(t), \quad \text { for all } t>0,
$$

and as a consequence, we get

$$
\limsup _{t \rightarrow+\infty}(p-1) \gamma^{2} \frac{\int_{0}^{t} \tilde{f}(s)^{2 \gamma-2} f^{\prime}(s)^{2} \mathrm{~d} s}{\tilde{f}(t)^{2 \gamma-1} f^{\prime}(t)} \leq \frac{(p-1) \gamma^{2}}{2 \gamma-1 /(p-1)} .
$$

We note that the right-hand side of this inequality is one for $\gamma=1 /(p-1)$. In this sense, hypothesis (21) is not very restrictive whenever (3) holds.

Finally, we have to mention that hypothesis $(p-2) /(p-1)<\tau_{-}$in our main results may be replaced by the weakest assumption (21) (see Lemma 12 below). However, for the sake of clarity, it seems better to consider $(p-2) /(p-1)<\tau_{-}$instead of $(21)$. We also note that in Proposition 10 it is not necessary to assume that $f$ is a $C^{2}$ function, but only $C^{1}$. Moreover, as a consequence of Proposition 10(i), one obtains that $u^{*}$ is bounded if $N<p+2 p /(p-1)$, since $\gamma \geq 1 /(p-1)$.

Proof of Proposition 10. Let $\tilde{f}(t)=f(t)-f(0), \lambda \in\left(0, \lambda^{*}\right)$, and let $u_{\lambda}$ be the minimal solution of $\left(1_{\lambda, p}\right)$. Recalling that $u_{\lambda} \in C^{1, \alpha}(\bar{\Omega})$ and the definition of $A_{u_{\lambda}}$ given in Definition 8, it is easy to check that $\psi:=\tilde{f}\left(u_{\lambda}\right)^{\gamma} \in A_{u_{\lambda}}$, since $\gamma \geq 1 /(p-1)$. Therefore, taking $\psi$ in the semi-stability condition (20), we obtain

$$
\lambda \int_{\Omega} \tilde{f}\left(u_{\lambda}\right)^{2 \gamma} f^{\prime}\left(u_{\lambda}\right) \mathrm{d} x \leq(p-1) \gamma^{2} \int_{\Omega} \tilde{f}\left(u_{\lambda}\right)^{2 \gamma-2} f^{\prime}\left(u_{\lambda}\right)^{2}\left|\nabla u_{\lambda}\right|^{p} \mathrm{~d} x .
$$

Let $g^{\prime}(t):=\tilde{f}(t)^{2 \gamma-2} f^{\prime}(t)^{2}$. Taking $\varphi=g\left(u_{\lambda}\right)$ as a test function in (4), we have

$$
\int_{\Omega} \tilde{f}\left(u_{\lambda}\right)^{2 \gamma-2} f^{\prime}\left(u_{\lambda}\right)^{2}\left|\nabla u_{\lambda}\right|^{p} \mathrm{~d} x=\lambda \int_{\Omega} \tilde{f}\left(u_{\lambda}\right) g\left(u_{\lambda}\right) \mathrm{d} x+\lambda f(0) \int_{\Omega} g\left(u_{\lambda}\right) \mathrm{d} x .
$$

From (24) and (25), we obtain

$$
\int_{\Omega} \tilde{f}\left(u_{\lambda}\right)^{2 \gamma} f^{\prime}\left(u_{\lambda}\right) \mathrm{d} x \leq(p-1) \gamma^{2}\left(\int_{\Omega} \tilde{f}\left(u_{\lambda}\right) g\left(u_{\lambda}\right) \mathrm{d} x+f(0) \int_{\Omega} g\left(u_{\lambda}\right) \mathrm{d} x\right) .
$$

Using (21) and (23), we obtain that

$$
\limsup _{t \rightarrow+\infty}(p-1) \gamma^{2} \frac{\tilde{f}(t) g(t)}{\tilde{f}(t)^{2 \gamma} f^{\prime}(t)}<1
$$

and

$$
\lim _{t \rightarrow+\infty} \frac{g(t)}{\tilde{f}(t)^{2 \gamma} f^{\prime}(t)}=0 .
$$

From these limits and (26), it follows that

$$
\int_{\Omega} \tilde{f}\left(u_{\lambda}\right)^{2 \gamma} f^{\prime}\left(u_{\lambda}\right) \mathrm{d} x \leq C,
$$

where $C$, here and in the rest of the proof, is a constant independent of $\lambda$. Moreover, by (22), we obtain

$$
\int_{\Omega} \frac{\tilde{f}\left(u_{\lambda}\right)^{2 \gamma+1}}{u_{\lambda}} \mathrm{d} x \leq C,
$$


and hence, since $f(t)^{1 /(p-1)}$ is superlinear at infinity by assumption $(2), f\left(u_{\lambda}\right)$ is uniformly bounded in $L^{2 \gamma+1-1 /(p-1)}(\Omega)$.

If $N<(2 \gamma+1-1 /(p-1)) p$ then, by Lemma 5(i), $u_{\lambda}$ is uniformly bounded in $L^{\infty}(\Omega)$. Therefore $u^{*}=\lim _{\lambda \uparrow \lambda^{*}} u_{\lambda}$ is a regular extremal solution of $\left(1_{\lambda^{*}, p}\right)$. This proves part of assertion (i).

Assume $N \geq(2 \gamma+1-1 /(p-1)) p$. Using Lemma 5(ii)-(iii), we have that $u_{\lambda}$ is uniformly bounded in $L^{r}(\Omega)$ for all

$$
1 \leq r<r_{0}:=\frac{(p-1)(2 \gamma+1-1 /(p-1)) N}{N-(2 \gamma+1-1 /(p-1)) p} .
$$

We note that $r_{0} \geq p$ since $\gamma \geq 1 /(p-1)$.

We will do an iterative process starting with $r_{0}$. Assume that there exists $r_{n} \geq p$ such that $u_{\lambda}$ is uniformly bounded in $L^{r}(\Omega)$ for all $1 \leq r<r_{n}$. Let

$$
\alpha_{n}:=\frac{2 \gamma+1}{1+r_{n}}
$$

and set $\Omega=\Omega_{1} \cup \Omega_{2}$, where

$$
\Omega_{1}:=\left\{x \in \Omega: \tilde{f}\left(u_{\lambda}\right)^{2 \gamma+1} / u_{\lambda}>\tilde{f}\left(u_{\lambda}\right)^{2 \gamma+1-\alpha_{n}}\right\}
$$

and

$$
\Omega_{2}:=\left\{x \in \Omega: \tilde{f}\left(u_{\lambda}\right) \leq u_{\lambda}^{1 / \alpha_{n}}\right\}
$$

From (27) we have

$$
\int_{\Omega_{1}} \tilde{f}\left(u_{\lambda}\right)^{2 \gamma+1-\alpha_{n}} \mathrm{~d} x \leq C .
$$

On the other hand,

$$
\int_{\Omega_{2}} \tilde{f}\left(u_{\lambda}\right)^{r} \mathrm{~d} x \leq \int_{\Omega_{2}} u_{\lambda}{ }^{\frac{r}{\alpha_{n}}} \mathrm{~d} x \leq C, \quad \text { for all } 1 \leq r<\alpha_{n} r_{n} .
$$

Therefore,

$$
f\left(u_{\lambda}\right) \in L^{r}(\Omega), \quad \text { for all } 1 \leq r<(2 \gamma+1) \frac{r_{n}}{1+r_{n}}=2 \gamma+1-\alpha_{n}=\alpha_{n} r_{n} .
$$

Using Lemma 5 again, the following assertions hold:

1. If $\left(1+r_{n}\right) N<(2 \gamma+1) r_{n} p$ then $u_{\lambda}$ is uniformly bounded in $L^{\infty}(\Omega)$. As a consequence, $u^{*}=\lim _{\lambda \uparrow \lambda^{*}} u_{\lambda}$ is a solution of $\left(1_{\lambda^{*}, p}\right)$.

2. If $\left(1+r_{n}\right) N \geq(2 \gamma+1) r_{n} p$ then $u_{\lambda}$ is uniformly bounded in $L^{r}(\Omega)$, for all

$$
1 \leq r<r_{n+1}:=\frac{(p-1)(2 \gamma+1) r_{n} N}{\left(1+r_{n}\right) N-(2 \gamma+1) r_{n} p} .
$$

We start the bootstrap argument with $r_{0}$ given in (28). If $N<(2 \gamma+1) p$ then assertion 1 holds for some $n$, and hence, part (i) in the proposition follows. If $N \geq(2 \gamma+1) p$ then we obtain, by assertion 2, an increasing sequence with limit

$$
r_{\infty}=\frac{((p-1)(2 \gamma+1)-1) N}{N-(2 \gamma+1) p} .
$$


From this, assertion 2, and (29), it follows that

$$
u^{*} \in L^{q}(\Omega) \quad \text { for all } 1 \leq q<\frac{((p-1)(2 \gamma+1)-1) N}{N-(2 \gamma+1) p}=\tilde{q}_{0}
$$

and

$$
f\left(u^{*}\right) \in L^{q}(\Omega) \quad \text { for all } 1 \leq q<\frac{(2 \gamma+1-1 /(p-1)) N}{N-p /(p-1)}=\tilde{q}_{1},
$$

since all the estimates obtained for $u_{\lambda}$ and $f\left(u_{\lambda}\right)$ are independent of $\lambda$.

Finally, we prove that $u^{*}=\lim _{\lambda \uparrow \lambda^{*}} u_{\lambda}$ is a solution of $\left(1_{\lambda^{*}, p}\right)$. Using $\gamma \geq 1 /(p-1)$, we have

$$
\tilde{q}_{1}=\frac{(2 \gamma+1-1 /(p-1)) N}{N-p /(p-1)} \geq \frac{p^{*}}{p^{*}-1},
$$

where $p^{*}=N p /(N-p)$. Therefore, we obtain that $f\left(u_{\lambda}\right)$ converges to $f\left(u^{*}\right)$ as $\lambda \uparrow \lambda^{*}$ in $L^{p^{*} /\left(p^{*}-1\right)}(\Omega)$ and also in $W^{-1, p^{\prime}}(\Omega)$, since $L^{p^{*} /\left(p^{*}-1\right)}(\Omega) \subset W^{-1, p^{\prime}}(\Omega)$. The continuity of $\left(-\Delta_{p}\right)^{-1}$ from $W^{-1, p^{\prime}}(\Omega)$ to $W_{0}^{1, p}(\Omega)$ gives that $u_{\lambda}$ converges, strongly in $W_{0}^{1, p}(\Omega)$, to $u^{*}$ as $\lambda \uparrow \lambda^{*}$. Hence, we conclude that for each $\varphi \in W_{0}^{1, p}(\Omega)$,

$$
\begin{aligned}
\int_{\Omega}\left|\nabla u^{*}\right|^{p-2} \nabla u^{*} \cdot \nabla \varphi \mathrm{d} x & =\lim _{\lambda \uparrow \lambda^{*}} \int_{\Omega}\left|\nabla u_{\lambda}\right|^{p-2} \nabla u_{\lambda} \cdot \nabla \varphi \mathrm{d} x \\
& =\lim _{\lambda \uparrow \lambda^{*}} \lambda \int_{\Omega} f\left(u_{\lambda}\right) \mathrm{d} x=\lambda^{*} \int_{\Omega} f\left(u^{*}\right) \varphi \mathrm{d} x .
\end{aligned}
$$

\section{Proof of Theorems 1 and 2}

In order to prove Theorem 2 we need the following technical lemma.

Lemma 12. Assume (2) and (3). Let $\tau_{-}$be defined in (9). If $\tau_{-}>(p-2) /(p-1)$ then every

$$
\gamma \in\left(\frac{1}{p-1}, \frac{1+\sqrt{1-(p-1)\left(1-\tau_{-}\right)}}{p-1}\right)
$$

satisfies (21).

Proof. Let $\tau \in(0,1)$. We have the following equivalence:

$$
(p-1) \gamma^{2} \frac{\int_{0}^{t} \tilde{f}(s)^{2 \gamma-2} f^{\prime}(s)^{2} \mathrm{~d} s}{\tilde{f}(t)^{2 \gamma-1} f^{\prime}(t)}<\tau
$$

if and only if

$$
G_{\gamma, \tau}(t):=(p-1) \gamma^{2} \int_{0}^{t} \tilde{f}(s)^{2 \gamma-2} f^{\prime}(s)^{2} \mathrm{~d} s-\tau \tilde{f}(t)^{2 \gamma-1} f^{\prime}(t)<0 .
$$

We note that

$$
G_{\gamma, \tau}^{\prime}(t)=\left[(p-1) \gamma^{2}-\tau(2 \gamma-1)-\tau \frac{\tilde{f}(t) f^{\prime \prime}(t)}{f^{\prime}(t)^{2}}\right] \tilde{f}(t)^{2 \gamma-2} f^{\prime}(t)^{2} .
$$

Let $\epsilon_{0}:=\tau_{-}-(p-2) /(p-1)>0$ and note that for every $\epsilon \in\left(0, \epsilon_{0}\right)$ there exists $t_{0}=t_{0}(\epsilon)>0$ such that

$$
G_{\gamma, \tau}^{\prime}(t) \leq\left[(p-1) \gamma^{2}-\tau\left(2 \gamma-1+\tau_{-}-\epsilon\right)\right] \tilde{f}(t)^{2 \gamma-2} f^{\prime}(t)^{2}, \quad \text { for all } t \geq t_{0} .
$$


Noting that $\tau_{0}:=(p-1)\left(1-\tau_{-}+\epsilon\right)<1$ (for all $\left.\epsilon \in\left(0, \epsilon_{0}\right)\right)$, we obtain that

$$
(p-1) \gamma^{2}-\left(2 \gamma-1+\tau_{-}-\epsilon\right) \tau<0,
$$

for all $\tau \in\left(\tau_{0}, 1\right)$ and

$$
\gamma \in\left[\frac{\tau}{p-1}, \frac{\tau+\sqrt{\tau\left(\tau-(p-1)\left(1-\tau_{-}+\epsilon\right)\right)}}{p-1}\right) .
$$

Moreover, since $f(t)^{1 /(p-1)}$ is superlinear at infinity and (22), we have

$$
\lim _{t \rightarrow+\infty} \tilde{f}(t)^{2 \gamma-2} f^{\prime}(t)^{2}=+\infty, \quad \text { for all } \gamma \geq \frac{1}{p-1} .
$$

Now, using the last limit, (30) and (31), we obtain

$$
\lim _{t \rightarrow+\infty} G_{\gamma, \tau}^{\prime}(t)=-\infty
$$

for all $\epsilon \in\left(0, \epsilon_{0}\right), \tau \in\left(\tau_{0}, 1\right)$, and $\gamma$ satisfying (32). In particular,

$$
\lim _{t \rightarrow+\infty} G_{\gamma, \tau}(t)=-\infty
$$

for the same range of parameters. The result follows from the last limit and the equivalence given at the beginning of the proof, in view of the arbitrariness of $\epsilon$ and $\tau$.

As a consequence of Proposition 10 and Lemma 12 we prove Theorem 2.

Proof of Theorem 2. Assume $\tau_{-}>(p-2) /(p-1)$. By Lemma 12, every

$$
\gamma \in\left(\frac{1}{p-1}, \frac{1+\sqrt{1-(p-1)\left(1-\tau_{-}\right)}}{p-1}\right)
$$

satisfies (21). Therefore, $u^{*}$ is a solution of $\left(1_{\lambda^{*}, p}\right)$ by Proposition 10.

(i) If in addition $N<N(p)$, where $N(p)$ is defined in (11), then the boundedness of $u^{*}$ follows from Proposition 10(i) and the arbitrariness of $\gamma$.

(ii) If in addition $N \geq N(p)$, then Proposition 10(ii) and the arbitrariness of $\gamma$ give that $u^{*} \in L^{q}(\Omega)$, for all $1 \leq q<q_{0}$, and $f\left(u^{*}\right) \in L^{q}(\Omega)$, for all $1 \leq q<\bar{q}_{1}$, where

$$
q_{0}=\left(p+2 \sqrt{1-(p-1)\left(1-\tau_{-}\right)}\right) \frac{N}{N-N(p)}
$$

and

$$
\bar{q}_{1}=\left(p+2 \sqrt{1-(p-1)\left(1-\tau_{-}\right)}\right) \frac{N}{(p-1) N-p} .
$$

Let $\tilde{q}=\left(p^{*}\right)^{\prime}$ be defined in (19). Noting that $\bar{q}_{1} \leq N / p$ (since $N \geq N(p)$ ) and $\tilde{q}<\bar{q}_{1}$, we have $f\left(u^{*}\right) \in L^{q}(\Omega)$ for all $\tilde{q} \leq q<\bar{q}_{1} \leq N / p$. Therefore, by Lemma 6 , we obtain that $u^{*} \in W_{0}^{1, r}(\Omega)$ with $1 \leq r<(p-1) N \bar{q}_{1} /\left(N-\bar{q}_{1}\right)$. We conclude the proof by noting that the exponent $q_{1}$ given in (12) coincides with $(p-1) N \bar{q}_{1} /\left(N-\bar{q}_{1}\right)$. 
Now, we prove Theorem 1 as a corollary of Theorem 2.

Proof of Theorem 1. (i) Assume $f$ convex and $1<p<2$. Under these assumptions it is clear that

$$
\frac{p-2}{p-1}<0 \leq \tau_{-} .
$$

Therefore, from Theorem 2, we obtain that $u^{*}$ is a bounded solution of $\left(1_{\lambda^{*}, p}\right)$ if

$$
N<N(p)=p+\frac{2 p}{p-1}\left[1+\sqrt{1-(p-1)\left(1-\tau_{-}\right)}\right] .
$$

We conclude by noting that

$$
N(p) \geq H(p)=p+\frac{2 p}{p-1}[1+\sqrt{2-p}]>6,
$$

where $H$ is given in (8).

(ii) Assume $0<\tau_{-}$and $p=2$. By Theorem 2, we obtain that $u^{*}$ is a bounded solution of $\left(1_{\lambda^{*}, p}\right)$ if

$$
N<N(2)=2+4\left(1+\sqrt{\tau_{-}}\right) .
$$

The assertion and the theorem follow noting that $N(2)>6$.

\section{Proof of Theorems 3 and 4}

We start proving Theorem 3 as a consequence of Proposition 10 and Lemmas 12 and 9.

Proof of Theorem 3. Assume $\tau_{-}>(p-2) /(p-1)$ and let $N(p)$ be given in (11). If $N<N(p)$ then the assertion follows from Theorem 2(i). Thus, we may assume $N \geq N(p)$. It follows from Lemma 12 and Proposition 10 that $u^{*}$ is a solution of $\left(1_{\lambda^{*}, p}\right)$ and

$$
f\left(u^{*}\right) \in L^{q}(\Omega) \quad \text { for all } q<\overline{q_{1}}=\left(p+2 \sqrt{1-(p-1)\left(1-\tau_{-}\right)}\right) \frac{N}{(p-1) N-p} .
$$

By Lemma 9, we obtain that $u^{*} \in L^{\infty}(\Omega)$ if

$$
\left(1-\frac{p-1}{m}\right) N<p \bar{q}_{1}
$$

or equivalently, if (14) holds.

In order to prove Theorem 4, we need the following technical result that states a relation between assumptions (13) and $\tau_{+}<1$.

Lemma 13. Let $f$ be a positive $C^{2}$ function such that $f^{\prime}(t)>0$, for all $t>0$. Let $\tau_{+}$be given in (15). If $\tau_{+}<1$ then, for every $\epsilon \in\left(0,1-\tau_{+}\right)$, there exists a positive constant $c$ depending on $\epsilon$ such that

$$
f(t) \leq c(1+t)^{\frac{1}{1-(\tau+\epsilon)}}, \quad \text { for all } t \geq 0 .
$$


Proof. Let $\epsilon \in\left(0,1-\tau_{+}\right)$. By definition of $\tau_{+}$there exists $t_{0}=t_{0}(\epsilon)$ such that $\tilde{f}(t) f^{\prime \prime}(t) \leq$ $\left(\tau_{+}+\epsilon\right) f^{\prime}(t)^{2}$, for all $t \geq t_{0}$. Therefore

$$
\left[\ln f^{\prime}(t)\right]^{\prime}=\frac{f^{\prime \prime}(t)}{f^{\prime}(t)} \leq\left(\tau_{+}+\epsilon\right) \frac{f^{\prime}(t)}{\tilde{f}(t)}=\left(\tau_{+}+\epsilon\right)[\ln \tilde{f}(t)]^{\prime}, \quad \text { for all } t \geq t_{0} .
$$

Integrating the last expression with respect to $t$, we obtain

$$
\ln \left(\frac{f^{\prime}(t)}{f^{\prime}\left(t_{0}\right)}\right) \leq \ln \left(\frac{\tilde{f}(t)}{\tilde{f}\left(t_{0}\right)}\right)^{\tau_{+}+\epsilon}, \quad \text { for all } t>t_{0},
$$

or equivalently,

$$
\frac{f^{\prime}(t)}{\tilde{f}(t)^{\tau_{+}+\epsilon}}=\left(\frac{\tilde{f}(t)^{1-\left(\tau_{+}+\epsilon\right)}}{1-\left(\tau_{+}+\epsilon\right)}\right)^{\prime} \leq \frac{f^{\prime}\left(t_{0}\right)}{\tilde{f}\left(t_{0}\right)^{\tau_{+}+\epsilon}}, \quad \text { for all } t>t_{0} .
$$

Integrating again, we obtain

$$
f(t) \leq\left[\left(1-\left(\tau_{+}+\epsilon\right)\right) \frac{f^{\prime}\left(t_{0}\right)}{\tilde{f}\left(t_{0}\right)^{\tau_{+}+\epsilon}}\left(t-t_{0}\right)+\tilde{f}\left(t_{0}\right)^{1-\left(\tau_{+}+\epsilon\right)}\right]^{\frac{1}{1-\left(\tau_{+}+\epsilon\right)}}+f(0),
$$

for all $t \geq t_{0}$. The lemma follows easily from the last inequality.

Finally, we prove Theorem 4 as a consequence of the previous lemma, Theorems 2 and 3.

Proof of Theorem 4. Since $\tau_{-}>(p-2) /(p-1), u^{*}$ is a solution of $\left(1_{\lambda^{*}, p}\right)$ by Theorem 2 .

(i) Assume $\tau_{+}<1$. By Lemma 13, for every $\epsilon \in\left(0,1-\tau_{+}\right)$, there exists a positive constant $c$ (depending on $\epsilon$ ) such that

$$
f(t) \leq c(1+t)^{\frac{1}{1-(\tau+\epsilon)}}, \quad \text { for all } t \geq 0 .
$$

Therefore, from Theorem 3 with $m=1 /\left(1-\left(\tau_{+}+\epsilon\right)\right)$, it follows that $u^{*} \in L^{\infty}(\Omega)$ if

$$
N<\frac{p}{p-1}\left(1+\frac{p}{1-(p-1)\left(1-\left(\tau_{+}+\epsilon\right)\right)}+\frac{2 \sqrt{1-(p-1)\left(1-\tau_{-}\right)}}{1-(p-1)\left(1-\left(\tau_{+}+\epsilon\right)\right)}\right) .
$$

Hence, we obtain the assertion by the arbitrariness of $\epsilon$.

(ii) It is clear from Theorem 2(i).

(iii) We conclude the proof by noting that the right-hand side of inequalities (16) and (17) is bigger than or equal to $F(p)=p+4 p /(p-1)$.

\section{Acknowledgment}

The author would like to thank Xavier Cabré for useful conversations.

\section{References}

[1] A. Alvino, L. Boccardo, V. Ferone, L. Orsina, G. Trombetti, Existence results for nonlinear elliptic equations with degenerate coercivity, Ann. Mat. 182 (2003) 53-79.

[2] H. Brezis, T. Cazenave, Y. Martel, A. Ramiandrisoa, Blow up for $u_{t}-\Delta u=g(u)$ revisited, Adv. Differential Equations 1 (1996) 73-90.

[3] H. Brezis, J.L. Vázquez, Blow-up solutions of some nonlinear elliptic problems, Rev. Mat. Complut. 10 (1997) 443-469. 
[4] X. Cabré, A. Capella, M. Sanchón, Regularity of radial semi-stable solutions of reaction equations involving the $p$-Laplacian (in preparation).

[5] X. Cabré, M. Sanchón, Semi-stable and extremal solutions of reaction equations involving the $p$-Laplacian, Comm. Pure Appl. Anal. (in press).

[6] M.G. Crandall, P.H. Rabinowitz, Some continuation and variational methods for positive solutions of nonlinear elliptic eigenvalue problems, Arch. Ration. Mech. Anal. 58 (1975) 207-218.

[7] E. Di Benedetto, $C^{1+\alpha}$ local regularity of weak solutions of degenerate elliptic equations, Nonlinear Anal. 7 (1983) $827-850$.

[8] S. Eidelman, Y. Eidelman, On regularity of the extremal solution of the Dirichlet problem for some semilinear elliptic equations of the second order, Houston J. Math. 31 (2005) 957-960.

[9] A. Ferrero, On the solutions of quasilinear elliptic equations with a polynomial-type reaction term, Adv. Differential Equations 9 (2004) 1201-1234.

[10] T. Gallouët, F. Mignot, J.P. Puel, Quelques résultats sur le problème $-\Delta u=\lambda e^{u}$, C. R. Acad. Sci. Paris 307 (1988) 289-292.

[11] J. García-Azorero, I. Peral, On an Emden-Fowler type equation, Nonlinear Anal. 18 (1992) 1085-1097.

[12] J. García-Azorero, I. Peral, J.P. Puel, Quasilinear problemes with exponential growth in the reaction term, Nonlinear Anal. 22 (1994) 481-498.

[13] N. Grenon, $L^{r}$ estimates for degenerate elliptic problems, Potential Anal. 16 (2002) 387-392.

[14] D.D. Joseph, T.S. Lundgren, Quasilinear Dirichlet problems driven by positive sources, Arch. Ration. Mech. Anal. 49 (1973) 241-269.

[15] J. Kinnunen, S. Zhou, A boundary estimate for nonlinear equations with discontinuous coefficients, Differential Integral Equations 14 (2001) 475-492.

[16] J. Leray, J.L. Lions, Quelques résultats de Višik sur les problèmes elliptiques nonlinéaires par les méthodes de Minty-Browder, Bull. Soc. Math. France 93 (1965) 97-107.

[17] G.M. Lieberman, Boundary regularity for solutions of degenerate elliptic equations, Nonlinear Anal. 11 (1988) 1203-1219.

[18] Y. Martel, Uniqueness of weak extremal solutions of nonlinear elliptic problems, Houston J. Math. 23 (1997) $161-168$.

[19] F. Mignot, J.P. Puel, Sur une classe de problèmes non linéaires avec nonlinéarité positive, croissante, convexe, Comm. Partial Differential Equations 5 (1980) 791-836.

[20] G. Nedev, Regularity of the extremal solution of semilinear elliptic equations, C. R. Acad. Sci. Paris 330 (2000) 997-1002.

[21] M. Sanchón, Regularity of the extremal solution of some nonlinear elliptic problemes involving the $p$-Laplacian (submitted for publication).

[22] P. Tolksdorf, Regularity for a more general class of quasilinear elliptic equations, J. Differential Equations 51 (1984) 126-150.

[23] D. Ye, F. Zhou, Boundedness of the extremal solution for semilinear elliptic problems, Commun. Contemp. Math. 4 (2002) 547-558. 